\title{
Unexpected Symmetry in the Nodal Structure of the He Atom
}

\author{
Dario Bressanini ${ }^{1, *}$ and Peter J. Reynolds ${ }^{2, \dagger}$ \\ ${ }^{1}$ Dipartimento di Scienze Chimiche e Ambientali, Universita' dell'Insubria, via Lucini 3, 22100 Como, Italy \\ ${ }^{2}$ Physics Division, Army Research Office, Research Triangle Park, North Carolina 27709, USA
}

(Received 17 November 2004; published 6 September 2005)

\begin{abstract}
The nodes of even simple wave functions are largely unexplored. Motivated by their importance to quantum simulations of fermionic systems, we have found unexpected symmetries in the nodes of several atoms and molecules. Here, we report on helium. We find that in both ground and excited states the nodes have simple forms. In particular, they have higher symmetry than the wave functions they come from. It is of great interest to understand the source of these new symmetries. For the quantum simulations that motivated the study, these symmetries may help circumvent the fermion sign problem.
\end{abstract}

DOI: 10.1103/PhysRevLett.95.110201

Generally speaking, the calculation of electronic structure is concerned with the determination of either the wave function itself or properties determined from expectation values over the wave function squared. The nodes of the wave functions are largely ignored. Here, motivated by a simulational approach to electronic structure, where the nodes do play an important role [1], we take a systematic look at the nodes of the helium atom. In as simple a system as $\mathrm{He}$, where the electronic structure has been very well studied [2], and despite its apparent simplicity, the exact wave function is unknown, and progress in its determination has been slow. Nor have there been more than scattered looks at its nodal structure.

Determination of a wave function, by either expansion methods or variational optimization, by its very nature involves the positioning of amplitude where it is large. Such an approach cares little about getting the structure right in regions where the wave function is small. Thus the nodes in particular are not being optimized. In fact, to traditional electronic structure methods, nodes of the trial function are simply irrelevant. This might explain the general neglect of interest in nodes. Yet they are of great significance in the growing field of quantum Monte Carlo (QMC) simulations. QMC simulations are of growing importance as they are the most accurate method of obtaining information about many-body systems, and one that scales very well [1]. In QMC simulations, the nodal structure determines the volume of the $3 N$-dimensional space that is to be sampled, and errors in the nodes are errors in the boundary conditions and, hence, in the solution to the Schrödinger equation. The key point here is that exact knowledge of the nodes allows for an exact stochastic solution of the Schrödinger equation. But is not knowledge of the nodes inherently as difficult (or as we have just noted, possibly more so) as knowledge of the wave function?

Here we show that for the helium atom the answer is decidedly "no." That is, one can exactly specify nodes even when the wave function is unknown. That is particularly obvious for the $1{ }^{1} S$ ground state, for it has no nodes. (That is, we know the node trivially, though the wave function clearly is not trivial.) Since there is no node, QMC simu-
PACS numbers: $02.70 . S s, 03.65 . G e$

lations can solve this state exactly. As we show in this Letter, the nodes of the excited states of He also appear to be simple. Moreover, based on our ongoing work, it appears that this simplification may be more general, as we see evidence for it in other atoms and seemingly small molecules as well. If true, the implications are significant: on the one hand, it allows for exact simulations of fermionic systems when we can determine the nodes, and may open up a systematic way to perform fermion simulations when the nodes can be modeled; on the other hand, it may lead to deeper understanding of many-body wave functions.

General observations about the nodes. - The Pauli principle imposes a global constraint, requiring antisymmetry of the wave function defined in a $3 N$-dimensional space, where $N$ is the number of particles (here two). Implicitly this defines a $3 N-1$ dimensional nodal hypersurface. However, the obvious "points" compose the so-called "Pauli hyperplane" $\left\{x_{1}=x_{2}, y_{1}=y_{2}, z_{1}=z_{2}\right\}$ which is only a subset of dimensionality $(3 N-3)$ of the full node. The rest of the nodal surface is usually not determined by antisymmetry alone (though it can be constrained by other symmetries), but depends on the potential and eigenstate through the solution of the Schrödinger equation [3]. Henceforth we implicitly factor out the spin by treating the $\alpha$ and $\beta$ electrons separately. That is, we make a spin assignment and consider only the remaining spatial part of the wave function. The exact spatial eigenfunctions for the (infinitely massive) helium atom (and likewise for all other two-electron atomic ions) are functions of just six spatial coordinates. Thus, e.g., $\Psi_{n}(\mathbf{R})=\Psi_{n}\left(\bar{r}_{1}, \bar{r}_{2}\right)=$ $\Psi_{n}\left(x_{1}, y_{1}, z_{1}, x_{2}, y_{2}, z_{2}\right)$.

The first triplet state: $1 s 2 s 2^{3} S$. - This excited state has the surprising property that its exact node is analytically known, despite the fact that no exact expression is known for the wave function itself. The derivation of the node is quite simple. Although it is part of the lore of the QMC community [4], we derive the analytical form here, since this does not appear widely known outside this community.

States with $S$ symmetry $(L=0)$ are rotationally invariant, and can thus be described by three fewer spatial coordinates, Many different choices for these coordinates 
have been used in the literature. The most extensively used are the interparticle coordinates $r_{1}, r_{2}, r_{12}$, and the trivially related coordinates $r_{1}, r_{2}, \theta_{12}$. Hylleraas introduced the coordinates $s=r_{1}+r_{2} ; \quad t=r_{1}-r_{2} ; \quad u=r_{12} \quad$ [5]. Pekeris extended this idea introducing perimetric coordinates, which are a linear combination of all three of $r_{1}, r_{2}$, and $r_{12}$ [6]. Also frequently used is a hyperspherical coordinate system introduced by Fock [7].

We choose interparticle coordinates. Applying the Pauli principle, we can write $\Psi\left(r_{1}, r_{2}, r_{12}\right)=-\Psi\left(r_{2}, r_{1}, r_{12}\right)$, from which we infer $\Psi\left(r, r, r_{12}\right)=0$ or that the node is simply $r_{1}-r_{2}=0$. Note $r_{1}$ and $r_{2}$ are not vectors here. Thus the node occurs whenever both electrons are at the same distance (as each other) from the nucleus, regardless of their separation $r_{12}$ (or angle $\theta_{12}$ ).

Thus we have analytically the node of the exact wave function, without knowing the exact wave function itself. The above argument is equally valid for $a n y{ }^{3} S$ state of helium, thus all these states share this node. (Of course, higher excited states must have additional nodes as well, to enforce orthogonality with the first triplet $S$ state.) Since only symmetry considerations were used, and the potential energy function was not needed in the proof, this is also the node of all other two-electron atomic systems. This property is quite remarkable: e.g., the $\mathrm{Li}^{+} 2^{3} S$ wave function is very different from the $\mathrm{He} 2^{3} S$ wave function, yet they have the same nodal structure. A corollary is that the node of exactly soluble models, such as the one in which the interelectronic repulsion is turned off, are also the same.

It is known that using these coordinates the exact wave function is not separable: $\Psi\left(r_{1}, r_{2}, r_{12}\right) \neq \phi\left(r_{1}, r_{2}\right) \varphi\left(r_{12}\right)$. However, for the $2^{3} S$ state we see that we can decompose the exact wave function into a positive definite function and a function representing the node, $\Psi\left(r_{1}, r_{2}, r_{12}\right)=$ $N\left(r_{1}, r_{2}\right) \times e^{f\left(r_{1}, r_{2}, r_{12}\right)}$. Here $e^{f\left(r_{1}, r_{2}, r_{12}\right)}$ is the unknown positive definite, totally symmetric function, written as an exponential to emphasize its positivity, and $N\left(r_{1}, r_{2}\right)$ is an antisymmetric function, with $N(r, r)=0$, that we call a nodal function since it describes the node. By this definition, a nodal function is any function that, when equated to zero, implicitly describes the nodal hypersurface of the wave function. The functions in the above decomposition are clearly not unique: different functions $N$ might describe the same nodal structure. Nevertheless it is truly unexpected that the antisymmetric function $N$ can be chosen to be a function of only the two variables $r_{1}$ and $r_{2}$. This means that the exact wave function depends on the interparticle distance while its node does not. The nodal function $N$ belongs to a higher symmetry group than $\Psi$. In this sense, the node is more symmetric than the wave function. Rotating a single electron around the nucleus leaves the nodal function invariant, but not the wave function. It is also interesting that the node can be described so simply: by the solution of an algebraic equation of particle distances.

Approximate wave functions usually do not give the correct nodes [8-12], but if the node is completely deter- mined by symmetry, all trial functions respecting that symmetry will have the correct node. For the ${ }^{3} S$ He this is, indeed, the case. Consider the simplest functional form able to approximately describe the first triplet state, namely, $\Psi=f_{1}\left(r_{1}\right) f_{2}\left(r_{2}\right)-f_{2}\left(r_{1}\right) f_{1}\left(r_{2}\right)$. This form, regardless of the form of the functions $f_{i}$, has the exact node.

Although the wave function has physical significance only for positive values of interparticle distances, we investigated numerically the wave function behavior even for negative values of the variables, since this might reveal information on its analytical structure. We used an antisymmetrized linear expansion of correlated exponentials $e^{a r_{1}+b r_{2}+c r_{12}}$. Even with only a two-term expansion, a node in the unphysical region appears. Expanding the wave function in a Taylor series around the origin, to first order we obtain $N=\left[c_{0}+c_{1}\left(r_{1}+r_{2}\right)+c_{2} r_{12}+\cdots\right]\left(r_{1}-r_{2}\right)$. With no loss of generality, and in order to make meaningful comparisons between different expansions, we normalize the wave functions so that $c_{1}=1$. Numerically, for a five term expansion $c_{0}=-0.03$. Note that a negative $c_{0}$ is an indication of the presence of a spurious nodal surface close to the origin. The most accurate wave function we examined, a 14 term expansion with an energy less than a microHartree away from the exact energy, gives $c_{0}=0.0051$. In Fig. 1 we show a superimposed plot of the nodes for various values of the angle $\theta_{12}$ for, respectively, the 5 term and the 14 term function.

Our numerical results suggest that the node in the unphysical region is present also in the exact wave function (i.e., the wave function is zero for negative values of distance). Thus, at first order, the wave function expanded around the origin is $N=\left[c_{0}+c_{1}\left(r_{1}+r_{2}\right)\right]\left(r_{1}-r_{2}\right)$ where the coefficients $c_{0}$ and $c_{1}$ are independent of $r_{12}$. While for an approximate wave function $c_{0}$ is not zero, numerical exploration of approximate but accurate wave functions suggests that the true $c_{0}$ is, indeed, zero. That is, the two nodes cross exactly at the origin. This means that at first order, around the nucleus, the wave function node has the simple crossing structure $\left(r_{1}+r_{2}\right)\left(r_{1}-r_{2}\right)$. As we saw above with the antisymmetrized linear expansion of correlated exponentials, a generic approximate wave function does not have this property. Remarkably, the exact wave

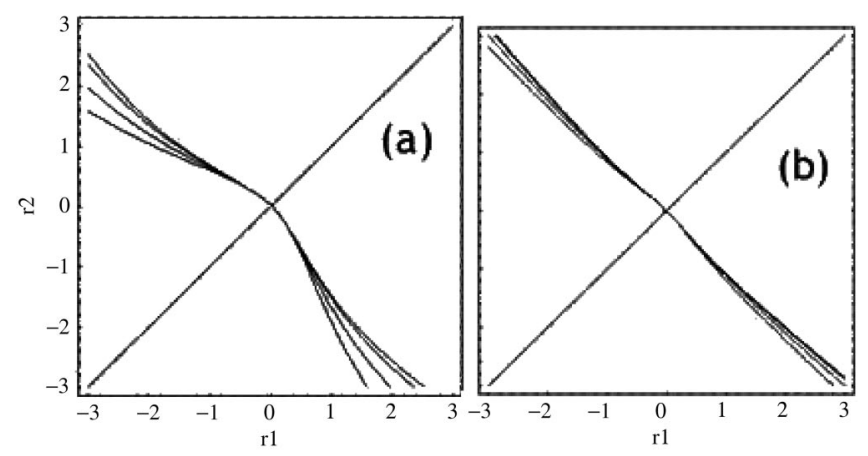

FIG. 1. Superimposed plots at various $\theta_{12}(0, \pi / 4, \pi / 2,3 \pi / 4)$ of $\Psi\left(r_{1}, r_{2}, \theta_{12}\right)=0$ for (a) 5 and (b) 14 term wave functions. 
function of the model system with the electron-electron interaction turned off, whose wave function is $\Psi=\left(r_{1}-\right.$ 1) $e^{-r_{1}-2 r_{2}}-\left(r_{2}-1\right) e^{-r_{2}-2 r_{1}}$, does have exactly this crossing node. Furthermore, it is easy to check that all two-electron atomic ions also have this property in this model. Given that the physical node $\left(r_{1}-r_{2}\right)$ does not contain $r_{12}$, it is tempting to expect that even the node in the unphysical region will not contain it.

$n p$ mp $P^{e}$ states. - In 1930 Breit [13] observed that for even-parity $P$ states of He, the exact wave function can be written as $\Psi=\left(x_{1} y_{2}-y_{1} x_{2}\right) \varphi\left(r_{1}, r_{2}, r_{12}\right)$. This is the solution with quantum number $L_{Z}=0$. If one desires a different symmetry axis, one simply replaces the term in parentheses by the component of $\bar{r}_{1} \times \bar{r}_{2}$ parallel to the axis of choice, where $\bar{r}_{1}$ and $\bar{r}_{2}$ are the position vectors of the two electrons.

Let us consider the first doubly excited state $2 p^{23} P^{e}$. Since $x_{1} y_{2}-y_{1} x_{2}$ is antisymmetric, the function $\varphi\left(r_{1}, r_{2}\right.$, $r_{12}$ ) must be symmetric with respect to the interchange of the two electrons and thus will not generate additional nodes. Higher ${ }^{3} P^{e}$ states will, of course, have additional nodes in $\varphi\left(r_{1}, r_{2}, r_{12}\right)$ to enforce orthogonality, but not antisymmetry. Once again the exact node is independent of $r_{12}$ while the wave function is not; and again we have the form of the node exactly but not that of the wave function.

For ${ }^{1} P^{e}$ states the spatial function $\Psi$ must be symmetric. The prefactor $x_{1} y_{2}-y_{1} x_{2}$ is antisymmetric, implying that $\varphi\left(r_{1}, r_{2}, r_{12}\right)$ must be antisymmetric. This necessarily means that we have the node $r_{1}=r_{2}$ by the same argument as for $\mathrm{He}^{3} S$. An exact factorization of the lowest energy ${ }^{1} P^{e}$ wave function is thus $\Psi=\left(x_{1} y_{2}-y_{1} x_{2}\right)\left(r_{1}-r_{2}\right) \times$ $\hat{\varphi}\left(r_{1}, r_{2}, r_{12}\right)$ where the symmetric function $\hat{\varphi}$ here in the singlet is different from the symmetric function $\varphi$ above in the triplet. We also note that the $r_{1}=r_{2}$ node is now present in a singlet state. Higher states would have additional nodes to impose orthogonality with lower states. And again we note the higher symmetry for the node.

Both singlet and triplet $P^{e}$ nodes contain $x_{1} y_{2}=x_{2} y_{1}$. Geometrically, this node occurs whenever the two electrons are both within a plane containing the $z$ axis (and hence also the nucleus), regardless of their interelectronic distances. We note, once again, the same node in different states. This is the behavior that one would expect from a separable system, although so far a factorization for the helium atom has not been found. (In a separable system $\Psi$ can be written as a product of terms, each of which can contain nodes in its coordinates. One or more of these functions would remain invariant in excited states, leading to a node that is present in many states.)

${ }^{1} P^{o}$ and ${ }^{3} P^{o}$ states. - In the same 1930 paper [13] Breit discussed ${ }^{1} P^{o}$ and ${ }^{3} P^{o}$ states. He showed that they could be described exactly by

$$
\begin{aligned}
& \Psi\left({ }^{1} P^{o}\right)=z_{1} f\left(r_{1}, r_{2}, r_{12}\right)+z_{2} f\left(r_{2}, r_{1}, r_{12}\right), \\
& \Psi\left({ }^{3} P^{o}\right)=z_{1} f\left(r_{1}, r_{2}, r_{12}\right)-z_{2} f\left(r_{2}, r_{1}, r_{12}\right) .
\end{aligned}
$$

These equations are not sufficient to completely fix the nodal structure, though they do describe a subset of the node in $3 N-2$ dimensions. In studying the $1 s 2 p^{3} P^{o}$ state of helium using a very accurate trial wave function, Anderson [14] found convincing numerical evidence that the full node can be described by the equation $g\left(r_{1}, z_{1}\right)=$ $g\left(r_{2}, z_{2}\right)$, indicating that the exact wave function is factorizable into $\left[g\left(r_{1}, z_{1}\right)-g\left(r_{2}, z_{2}\right)\right] \varphi\left(r_{1}, r_{2}, r_{12}\right)$. As recognized by Anderson, this is an unexpected higher symmetry since it is not required by Eqs. (1). While symmetry principles (angular momentum conservation and the Pauli principle) are clearly at play in the derivation of the nodal structure of the ${ }^{3} S$ and singlet and triplet $P^{e}$ states, as shown above, the result for the nodal structure of the ${ }^{3} P^{o}$ state does not have this origin. Thus if this node is correct, it must have a deeper, and still unknown, origin. It is likely, by analogy with the ${ }^{3} P^{o}$ state, that the $1 s 2 p^{1} P^{o}$ state could be described by $\left[g\left(r_{1}, z_{1}\right)+g\left(r_{2}, z_{2}\right)\right] \varphi\left(r_{1}, r_{2}, r_{12}\right)$, although to our knowledge this has not been checked yet.

$1 s 2 s^{1} S$ state. - We know of no previous work on the nodes of the first singlet $S$ state, i.e., the $1 s 2 s^{1} S$ state. This state must have a node to make the state orthogonal to the ground state, but there do not appear to be additional symmetry constraints to exploit. For the electronic structure of this state, various very accurate expansions using different coordinate systems have been explored (see Ref. [2]). With the structure of the node in mind, we examined a series of increasingly accurate Hylleraas expansions of the exact wave function using the MATHEMATICA ${ }^{\circledR}$ software package. An examination of the node of a 70 term expansion (whose energy is -2.145971069 a.u. compared to the exact value of -2.145974046 a.u.) is shown in Fig. 2. The figure shows practically no dependence on the interelectronic angle, which is plotted vertically.

Examining a longer expansion, with 203 terms, the energy improves to $E=-2.145973975$. The plot in the inset shows a superposition of cuts of the node of this latter trial function for different values of the angle $\left\{\theta_{12}=\right.$ $0, \pi / 6, \pi / 3, \ldots, \pi\}$. It shows even less deviation as a

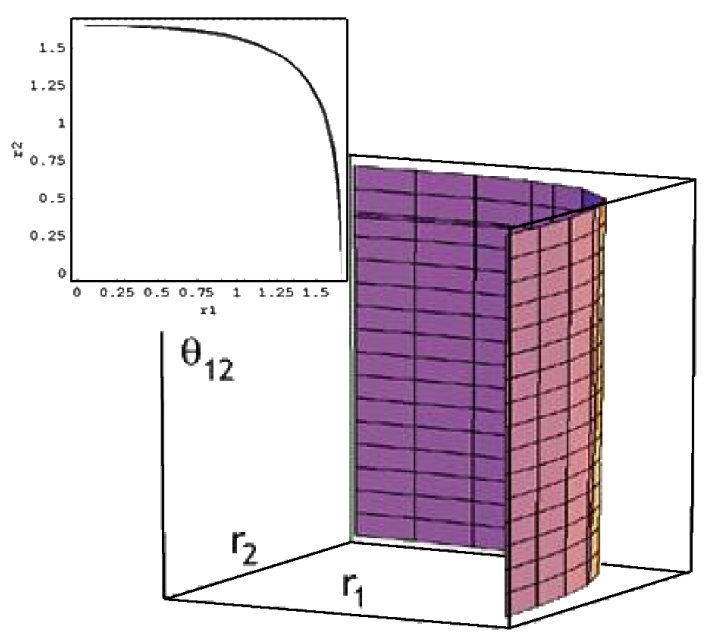

FIG. 2 (color online). Surface contour plot with inset of superimposed cuts at various $\theta_{12}$ of the node of 70 and 203 term He $1 s 2 s^{1} S$ wave functions. 


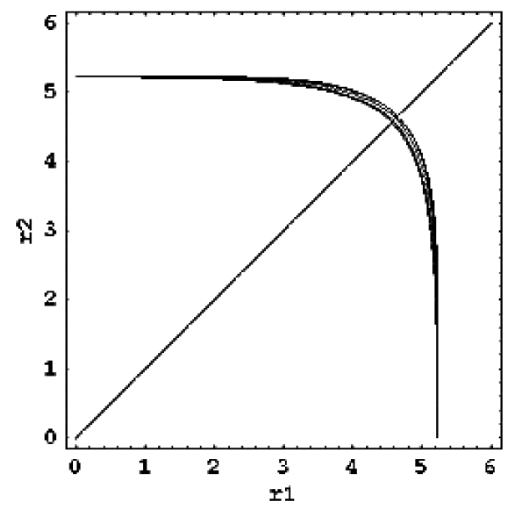

FIG. 3. Superimposed plots at various $\theta_{12}(0, \pi / 4, \pi / 2,3 \pi / 4)$ of $\Psi\left(r_{1}, r_{2}, \theta_{12}\right)=0$ for the 84 term He $1 s 3 s^{3} S$ wave function.

function of $\theta_{12}$. (Likewise, the deviation for shorter expansions becomes more noticeable.) Thus we see strong convergence toward a node with no $r_{12}$ dependence.

This fact is quite remarkable given that each term of the Hylleraas expansion is of the form $r_{1}^{n} r_{2}^{m} r_{12}^{k} e^{a r_{1}+b r_{2}+c r_{12}}$, properly symmetrized. To converge to a form independent of $r_{12}$ there must be a very delicate cancellation of terms when one is exactly on the node.

$1 s 3 s^{3} S$ state. - The second triplet $S$ state must be orthogonal to the lower lying triplet $S$ states, whose exact $\left(r_{1}=r_{2}\right)$ node we showed earlier. That node must still be present though, by the earlier argument. A plot of the node of an 84 term Hylleraas expansion is shown in Fig. 3.

We can see the $r_{1}-r_{2}$ node and, in addition, a structure similar to the one we just saw above for the second singlet $S$ state. The shape again seems "almost" independent of the interelectronic angle (distance) coordinate, and improves in "convergence" with expansion length.

Conclusions. - The nodes of the excited states of the helium atom quite generally appear to belong to higher symmetry groups than the wave functions they are embedded in. We have shown a number of exact nodal structures where there are no exact wave functions. Moreover, we seem to see a node independent of $r_{12}$ even in states for which we have only numerical results, and where the wave functions are not independent of that coordinate.

The ${ }^{1} S$ and ${ }^{3} S$ nodes are exactly of the topology implicitly generated in the hyperspherical treatment of the helium atom. There the shape of the various nodes come out as spherical, while numerically it appears that the nodes are actually quite similar, but deformed from that. The lower dimensional nodes (in $r_{1}$ and $r_{2}$ separately, for example) are spherical. But the "hyperspherical" coordinate appears to be (perhaps) a higher power combination of the individual coordinates. Specifically, a least squares fit shows that nodal surfaces of the $1 s 2 s^{1} S$ and $1 s 3 s^{3} S$ states seem to be, respectively, $r_{1}^{4}+r_{2}^{4}=$ const and $r_{1}^{5}+r_{2}^{5}=$ const. At this point, however, we would have to consider these forms no more than numerical fits.

We also note a striking similarity between the shape of the ${ }^{1} S$ and ${ }^{3} S$ nodes and the first unstable periodic orbit of the semiclassical treatment of $\mathrm{He}$ [15]. In semiclassical treatments, stable periodic orbits give regions of high probability, while unstable orbits give regions of low probability. Thus we can consider the latter orbits as the semiclassical nodes. If so, the orbits in Fig. 15 of Ref. [15] give a striking confirmation of the nodes we have seen.

While we examined only the helium atom here, there are indications that some of the properties we describe might be present also in many-electron atoms. For example, in Ref. [8] and in ongoing work by the present authors, there is numerical evidence that the node of the lithium atom appears to have higher symmetry than its wave function, and possibly of a trivial form. In Ref. [16] Mitas and coworkers investigate the nodal properties of spin-polarized states of few-electron ions and molecules and also find simplifications. Further work is needed to clarify whether these are general properties of nodes of yet larger atomic and molecular systems.

This work was carried out in part at the Physics Department of Georgetown University. We thank them for their gracious hospitality.

*Electronic address: Dario.Bressanini@uninsubria.it

${ }^{\dagger}$ Also at Department of Physics, Georgetown University, Washington, DC, USA.

Electronic address: Peter.Reynolds@us.army.mil

[1] B. L. Hammond, W. A. Lester, Jr., and P. J. Reynolds, Monte Carlo Methods in ab initio Quantum Chemistry (World Scientific, Singapore, 1994).

[2] R. C. Forrey, Phys. Rev. A 69, 022504 (2004), and references therein.

[3] D. M. Ceperley, J. Stat. Phys. 63, 1237 (1991).

[4] D. J. Klein and H. M. Pickett, J. Chem. Phys. 64, 4811 (1976).

[5] E. A. Hylleraas, Z. Phys. 54, 347 (1929).

[6] C. L. Pekeris, Phys. Rev. 112, 1649 (1958).

[7] V. A. Fock, Izv. Akad. Nauk SSSR, Ser. Fiz. 18, 161 (1954).

[8] W. A. Glauser, W. R. Brown, W. A. Lester, Jr., D. Bressanini, B. L. Hammond, and M.L. Koszykowski, J. Chem. Phys. 97, 9200 (1992).

[9] D. Bressanini, D. M. Ceperley, and P.J. Reynolds, in Recent Advances in Quantum Monte Carlo Methods, edited by W. A. Lester, S. M. Rothstein, and S. Tanaka (World Scientific, Singapore, 2002), 2nd ed.

[10] R. N. Barnett, P. J. Reynolds, and W. A. Lester, Jr., J. Chem. Phys. 82, 2700 (1985).

[11] P. J. Reynolds, R. N. Barnett, and W. A. Lester, Jr., Int. J. Quantum Chem., Quantum Chem. Symp. 18, 709 (1984).

[12] J. Hachmann, P. T. A. Galek, T. Yanai, G. K. L. Chan, and N. C. Handy, Chem. Phys. Lett. 392, 55 (2004).

[13] G. Breit, Phys. Rev. 35, 569 (1930).

[14] J. B. Anderson, Phys. Rev. A 35, 3550 (1987).

[15] G. Tanner, K. Richter, and J.-M. Rost, Rev. Mod. Phys. 72, 497 (2000).

[16] M. Bajdich, L. Mitas, G. Drobny, and L. K. Wagner, condmat/0409406 [Phys. Rev. B (to be published)]. 